\title{
Congestion-free embedding of $2(n-k)$ spanning trees in an arrangement graph is
}

\author{
Yuh-Shyan Chen ${ }^{\mathrm{a}, *}$, Tong-Ying Juang ${ }^{\mathrm{a}}$, Ying-Ying Shen ${ }^{\mathrm{b}}$ \\ a Department of Statistics, National Taipei University, Taipei 10433, Taiwan \\ b Optoma Corporation, Hsin-Chu, Taiwan
}

Received 25 October 1999; received in revised form 10 June 2000; accepted 1 September 2000

\begin{abstract}
The arrangement graph $A_{n, k}$ is not only a generalization of star graph $(n-k=1)$, but also more flexible. In this investigation, we elucidate the problem of embedding of multiple spanning trees in an arrangement graph with the objective of congestion-free. This result is to report how to exploit $2(n-k)$ edge disjoint spanning trees in an arrangement graph, where each congestion-free spanning tree's height is $2 k-1$. Our scheme is based on a subgraphpartitioning scheme. First, we construct $2(n-k)$ base spanning trees in every $A_{n-k+2,2}$. Then, we recursively construct $2(n-k)$ spanning trees from every $A_{n-k+2,2}$ up to $A_{n, k}$ by a bottom-up approach. This is a near-optimal result since all of possible edges in the base subarrangement $A_{n-k+2,2}$ are fully utilized. (C) 2001 Published by Elsevier Science B.V. All rights reserved.
\end{abstract}

Keywords: Arrangement graph; Embedding; Interconnection network; Parallel processing; Spanning tree

\section{Introduction}

Designing large multi-processor systems frequently involves organizing into various configurations. One of the widely studied interconnection network topologies is the star graph $[10,12,13]$. As a member of the Cayley graphs, the star graph possesses several attractive features such as its diameter-to-node-degree ratio, scalability, partitionability, symmetry, and high degree of fault tolerance [1,4]. However, the star graph is limited with respect to its number of nodes: $n$ ! for an $n$-dimensional star graph. A new interconnection topology, arrangement graph, has recently been proposed [5]. As a family of undirected graphs that contains the star graph family, the arrangement graph has desired properties, such as symmetric vertex and symmetric edge, strong resilience and maximal fault-tolerance. Arrangement graph is more flexible than the star graph in terms of choosing major design parameters, i.e. member of vertices, degree and diameter, while preserving most of the excellent properties of the star graph.

\footnotetext{
A preliminary version of this paper is presented at National Computer Symposium (NCS), Taiwan, ROC, 1999, and was supported by the National Science Council, ROC, under Contract NSC89-2213-E-216-010 and NSC89-2213-E-305-001.

* Corresponding author.

E-mail addresses: yschen@mail.ntpu.edu.tw (Y.-S. Chen), ying@optoma.com.tw (T.-Y. Juang).
} 
The arrangement graph has received considerable attention in [2,5-9,11]. First, Day and Tripathi [6] designed a shortest-path routing algorithm for the arrangement graphs. According to their results, the arrangement graph can be embedded cycles whose length ranges from three to the size of the graph [7]. Moreover, the arrangement graph can be decomposed into vertex disjoint cycles in many different ways [7]. Furthermore, multi-dimensional graphs, hypercube and one spanning tree can be embedded in arrangement graph [8]. The spanning tree can support broadcasting communication in the arrangement graph. Tsai and Horng proposed an efficient scheme to embed hypercube on arrangement graphs [11]. Heieh and Chen [9] further demonstrated that the arrangement graph remains a ring even if it is faulty. Bat et al. [2] recently proposed a distributed fault-tolerant algorithm for one-to-all broadcasting only in the one-port communication model on the arrangement graph.

In the light of above discussion, this work elucidates the problem of construction of multiple spanning trees in arrangement graphs. In this investigation, we assume that a node consists of a processor with bidirectional communication links to each of its adjacent nodes. Therefore, the term edge-disjoint spanning trees can be interchangeably adopted to reflect that no two edges of our spanning trees share a same direct communication link. To our knowledge, this work reports to the feasibility of embedding $2(n-k)$ spanning trees in $A_{n, k}$, at the same time, keeping the edge-congestion free. Similar results for the star graph can be found in $[3,14]$.

A tree is a common structure to represent inter-task communication pattern of a parallel algorithm. In this work, we propose a new spanning tree in an arrangement graph $A_{n, k}$ that has nice property that $2(n-k)$ copies of such trees can be embedded simultaneously in the network with edge-congestion free. In this paper, we consider the embedding of multiple spanning trees in an arrangement graph with the objective of congestion free. Chen et al. recently proposed a scheme to embed $n-k$ spanning trees [3]. Further, this paper exploits the double number of spanning trees than Chen et al.'s scheme.

The rest of this paper is organized as follows. Section 2 introduces preliminaries. Section 3 presents the scheme of embedding $2(n-k)$ spanning trees. Conclusions are finally drawn in Section 4.

\section{Preliminaries}

The arrangement graph is denoted by $A_{n, k}$, where specified by integers $n$ and $k$ and $1 \leqslant k \leqslant n-1$. Denote $\langle n\rangle=\{1,2, \ldots, n\}$. Let $P\left(\begin{array}{l}n \\ k\end{array}\right)$ be the set of permutations of $k$ symbols taken from $\langle n\rangle$. These $k$ symbols are denoted as $X=x_{1} x_{2} \cdots x_{k}$. Refer $x_{i}$ as the $i$ th element of $X$. The $(n, k)$-arrangement graph, denoted as $A_{n, k}$, defined in [6] is an undirected graph $(V, E)$ as follows:

$$
\left\{\begin{array}{l}
V=\left\{X=x_{1} x_{2} \cdots x_{k} \mid x_{i} \text { in }\langle n\rangle \text { and } x_{i} \neq x_{j} \text { for } i \neq j\right\}=P\left(\begin{array}{l}
n \\
k
\end{array}\right), \\
E=\left\{(x, y) \mid x \text { and } y \text { in } V \text { and for some } i \text { in }\langle k\rangle, x_{i} \neq y_{j} \text { and } x_{j}=y_{j} \text { for } j \neq i\right\} .
\end{array}\right.
$$

Fig. 1 depicts an example of $A_{4,2}$ and $A_{5,3}$. The edge of $A_{n, k}$ connecting neighboring nodes, which differ in exactly one of their $k$ positions. The vertices of $A_{n, k}$ are the arrangements of $k$ elements of $\langle n\rangle$. For example, in $A_{4,2}$, the node $p=41$ is connected to the nodes $=42,43,21$ and 31 . An edge of $A_{n, k}$ connecting two arrangements $p$ and $q$, which differ only in position $i$, is called an $i$-edge. For all values of $n$ and $k, A_{n, k}$ is a regular graph on $n ! /(n-k)$ ! nodes that is regular of degree $k(n-k)$, and a diameter $\lfloor 3 / 2 k\rfloor[6]$. For an arrangement $X=x_{1} x_{2} \cdots x_{k}$, we define $\operatorname{EXT}(X)=\langle n\rangle-\left\{x_{1}, x_{2}, \ldots, x_{k}\right\}$ to be the $n-k$ elements of $\langle n\rangle$ not appearing in the arrangement $X$. Let $I N T(X)=\left\{x_{1}, x_{2}, \ldots, x_{k}\right\}=\langle n\rangle-E X T(x)$ to be the $k$ elements of $\langle n\rangle$ appearing in the arrangement $X$. For example, we consider the node $p=412$ in the arrangement graph $A_{5,3}$, so $\operatorname{EXT}(p)=\{3,5\}$ and $\operatorname{INT}(p)=\{1,2,4\}$.

In an $A_{n, k}$, each node $p$ performs an adjacent function $A D J_{x, y}(p)$ to arrive at adjacent node $q$, where $x$ is the position of label of node and $y$ is the changed label in $\operatorname{EXT}(p)$. Given $X=x_{1} \cdots x_{x} \cdots x_{k} \in I N T(X)$, the 


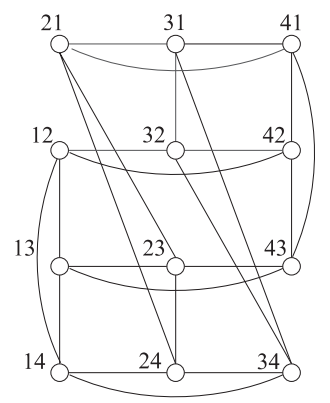

(a)

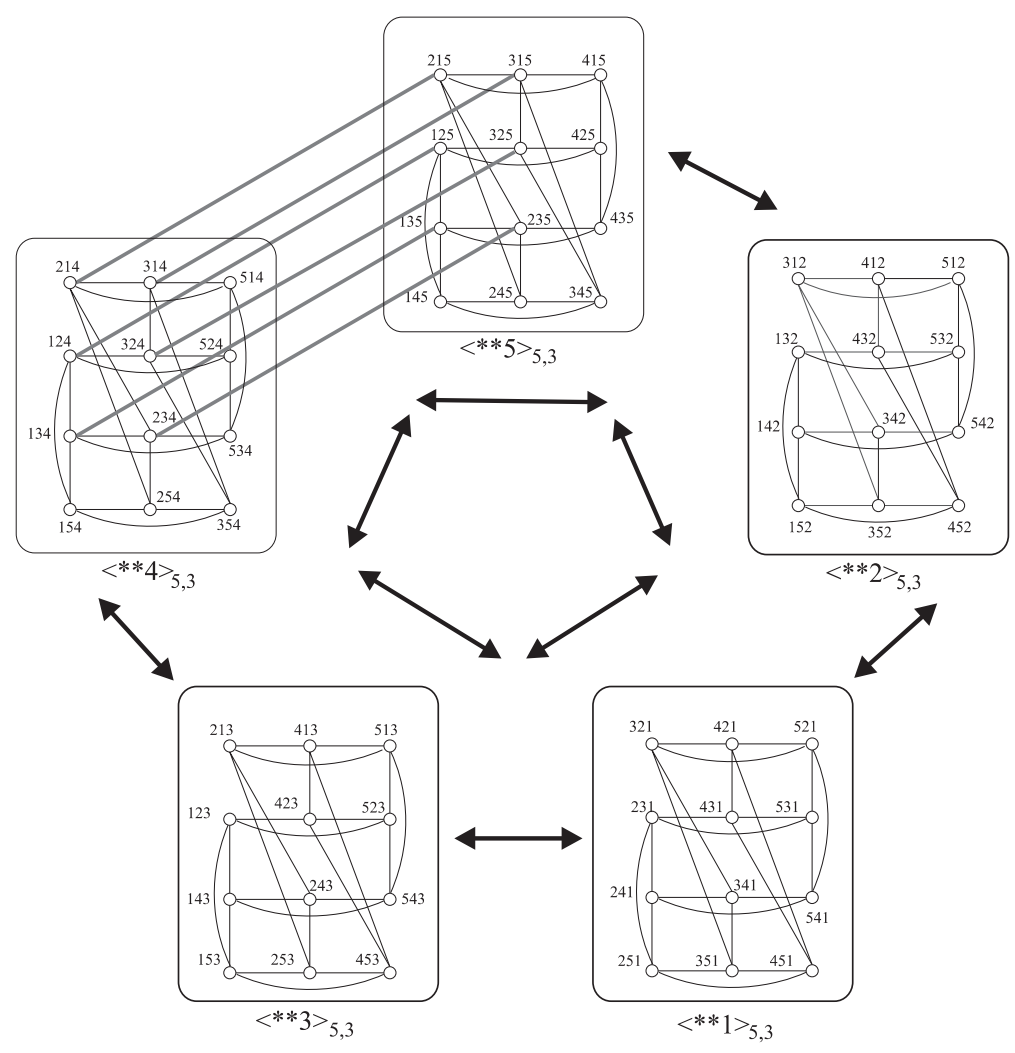

(b)

Fig. 1. The example for arrangement graph: (a) $A_{4,2}$; (b) $A_{5,3}$.

adjacent function $A D J_{x, y}(X)$ is the adjacent nodes of $X$ obtained by changing $x_{x}$ in $X$ as $y$, where $1 \leqslant x \leqslant k$ and $y \in E X T(X)$. Consider an arrangement graph $A_{5,3}$, adjacent nodes for node $p=412$ are 413, 415, 432, 452, 312 and 512, where $\operatorname{EXT}(p)=\{3,5\}, I N T(p)=\{1,2,4\}, A D J_{1,3}(p)=312, A D J_{1,5}(p)=512, A D J_{2,3}(p)=$ $432, A D J_{2,5}(p)=452, A D J_{3,3}(p)=413$, and $A D J_{3,5}(p)=415$.

The $A_{n, k}$ is with recursive structure [6] i.e., an $A_{n, k}$ can be partitioned into $n$ copies of $A_{n-1, k-1 \text {, each }}$ embedded $A_{n-1, k-1}$ is conveniently denoted by $\left\langle *^{k-1} \alpha\right\rangle_{n, k}$, where $\alpha \in\{1,2, \ldots, n\}$, (where $*$ represents a 
"don't care" symbol). For example, $\langle * * 3\rangle_{4,3}$ represents an embedded $A_{3,2}$ of $A_{4,3}$, contains six nodes: 123 , $143,213,243,413$, and 423. From other point of view, there are $n$ copies of $\left\langle *^{k-1} \alpha\right\rangle_{n, k}$, which are obtained by performing a split operation on $\left\langle *^{k}\right\rangle_{n, k}$, where $\alpha \in\{1,2, \ldots, n\}$. This operation is called $k$-partition. Generally, $A_{n, k}$ can be partitioned into $n ! /(n-p)$ ! node-disjoint copies of $A_{n-p, k-p}$ in $n ! /(p !(n-p) !)$ different ways and that in total $A_{n . k}$ contains $\left(\begin{array}{l}k \\ p\end{array}\right)(n !) /((n-p) !)$ copies of $A_{n-p, k-p}$, for $1 \leqslant p \leqslant k-1$.

As a conclusion, the drawback of star graph $[10,12,13]$ is limited with respect to its number of nodes, which is equal to $n !$. This implies what the low scalability will be. The proposed arrangement graph [6] is motivated by having the high scalability. The arrangement graph is more flexible than the star graph in terms of choosing major design parameters, i.e. member of vertices, degree and diameter, while preserving most of the excellent properties of the star graph. However, the potential troubles of the arrangement graph [6] is the high-degree problem, which is $k(n-k)$. This problem leads to troublesome development of a VLSI layout for the real machine.

\section{Congestion-free embedding of $2(n-k)$ spanning trees}

This section presents a novel embedding scheme of embedding $2(n-k)$ edge-disjoint spanning trees. Our construction scheme adopts a bottom-up manner. An $A_{n, k}$ can be partitioned into $n ! /(n-k+2)$ ! copies of $A_{n-k+2,2}$. Each $A_{n-k+2,2}$ initially construct $2(n-k)$ base spanning trees. For each $A_{n-k+3,3}$, there are $n-k+3$ copies of $A_{n-k+2,2}$. Each $A_{n-k+3,3}$ will perform a concatenation operation among $n-k+3$ copies of $A_{n-k+2,2}$ to construct $2(n-k)$ spanning trees in the $A_{n-k+3,3}$. Recursively performing the concatenation operations allow us to finally construct $2(n-k)$ spanning trees in an $A_{n, k}$.

Our $2(n-k)$ spanning trees are constructed by two phases:

- Phase 1. Generate $2(n-k)$ base spanning trees in each $A_{n-k+2,2}$.

- Phase 2. Perform a recursive concatenation operation to embed $2(n-k)$ edge-disjoint spanning trees.

These phases are described as follows.

\subsection{Phase 1: Generate $2(n-k)$ base spanning trees in an $A_{n-k+2,2}$}

After a splitting-operation on $A_{n, k}, n ! /((n-k+2) !)$ copies of $A_{n-k+2,2} \mathrm{~s}$ are obtained. According to different values of $n$ and $k, 2(n-k)$ base spanning trees in each $A_{n-k+2,2}$ can be generated as follows:

- Step 1. Locate $2(n-k)$ roots nodes.

- Step 2. Generate $2(n-k)$ base spanning trees in each $A_{n-k+2,2}$.

\subsubsection{Step 1. Locating $2(n-k)$ roots in an $A_{n-k+2,2}$}

This section describes how to locate $2(n-k)$ root nodes in an $A_{n-k+2,2}$. An $A_{n-k+2,2}$ is partitioned into $n-k+2$ copies of $A_{n-k+1,1}$, where each $A_{n-k+1,1}$ is a $(n-k+1)$-node complete graph. The $S W P_{1,2}(R)$ function is defined to swap the first and second bits of $R$. Denote these $2(n-k)$ root nodes as $R_{1}, R_{2}, \ldots, R_{n-k}$ and $P_{1}, P_{2}, \ldots, P_{n-k}$, which are constructed as follows:

- Let $R_{1}=\left(y_{1} x_{2} x_{3} \cdots x_{k}\right)$ be any node of one $A_{n-k+1,1}$. Other root nodes $R_{2}=\left(y_{2} x_{2} x_{3} \cdots x_{k}\right)$, $R_{3}=\left(y_{3} x_{2} x_{3} \cdots x_{k}\right), \ldots$, and $R_{n-k+1}=\left(y_{n-k+1} x_{2} x_{3} \cdots x_{k}\right)$ by exchanging the first bit of $R_{1}$ with $\alpha$, where $\alpha \in \operatorname{EXT}\left(R_{1}\right)=\left\{y_{2}, \ldots, y_{n-k+1}\right\}$. This work is achieved as follows:

$R_{i}=A D J_{1, \alpha}\left(R_{1}\right)$, where $2 \leqslant i \leqslant n-k+1$ and $\alpha \in E X T\left(R_{1}\right)$.

Clearly, each pair of $R_{1}, R_{2}, \ldots$, and $R_{n-k+1}$ are adjacent since $R_{1}, R_{2}, \ldots$, and $R_{n-k+1}$ belong to an $A_{n-k+1,1}$ (a complete graph $\left(* x_{2} x_{3} \cdots x_{k}\right)$ ). Note that node $R_{n-k+1}$ is used to be a template node to ensure congestion-free in our embedding. 
- Let $P_{i}=S W P_{1,2}\left(R_{i}\right)$, where $1 \leqslant i \leqslant n-k+1$. Note that $P_{1}, P_{2}, \ldots$, and $P_{n-k+1}$ also belong to a distinct $A_{n-k+1,1}$ (a complete graph $\left(x_{2} * x_{3} \cdots x_{k}\right)$ ). Similarly node $P_{n-k+1}$ is used to be a template node to ensure congestion-free in our embedding.

Intuitively, every $R_{i}$ is only different in the first bit, and every $P_{i}$ is only different in the second bit. Root nodes $R_{i}, 1 \leqslant i \leqslant n-k$, are selected from $\left(* x_{2} x_{3} \cdots x_{k}\right)$. Other root nodes $P_{i}, 1 \leqslant i \leqslant n-k$, are selected from $\left(x_{2} * x_{3} \cdots x_{k}\right)$. Clearly, both $\left(* x_{2} x_{3} \cdots x_{k}\right)$ and $\left(x_{2} * x_{3} \cdots x_{k}\right)$ are $A_{n-k+1,1}$ or $(n-k+1)$-node complete graph. Note that root nodes $R_{1}, R_{2}, \ldots, R_{n-k}$ and $P_{1}, P_{2}, \ldots, P_{n-k}$ satisfy the following property.

Root-location property. There are $2(n-k)$ root nodes $R_{i}$ and $P_{i}, 1 \leqslant i \leqslant n-k$, in same subarrangement graph. Root nodes $R_{i}, i=1, \ldots, n-k$, belong to an $A_{n-k+1,1}, P_{i}, i=1, \ldots, n-k$, belong to a distinct $A_{n-k+1,1}$, so $P_{i}=\operatorname{SWP}_{1,2}\left(R_{i}\right)$, for $i=1, \ldots, n-k$.

Root nodes $R_{i}$ and $P_{i}, i=1, \ldots, n-k$, are used to expand $2(n-k)$ base spanning trees in an $A_{n-k+2,2}$. Fig. 2 illustrates an example by setting $R_{1}=21, R_{2}=31$ and $P_{1}=12, P_{2}=13$. Node $R_{3}=41$ and $P_{3}=14$ are the template nodes. This root-location property is very useful during constructing $2(n-k)$ spanning trees.

\subsubsection{Step 2. Generate $2(n-k)$ base edge-disjoint spanning trees in an $A_{n-k+2,2}$}

The $2(n-k)$ base spanning trees are constructed from root $R_{i}$ and $P_{i}, 1 \leqslant i \leqslant n-k$, in an $A_{n-k+2,2}$. The fact that each $A_{n-k+1,1}$ is a complete graph accounts for why $2(n-k)$ base spanning trees can be constructed if we can connect each root node to distinct node of all other $A_{n-k+1,1} \mathrm{~s}$. This is because we have the following result and Fig. 3 gives an example.

Lemma 1. For a $\kappa$-node complete graph $G$, there exist $\kappa$ disjoint spanning trees with height one. Notably, each node in $G$ is the root node of each spanning tree.

Now we explain how to construct $2(n-k)$ base spanning trees in each $A_{n-k+2,2}$ such that each tree's height is 3 . Given $2(n-k)$ root nodes $R_{i}$ and $P_{i}, 1 \leqslant i \leqslant n-k$. Consider any pair of nodes $R \in R_{i}$ and $P \in P_{i}$, where $R=\operatorname{SWP}_{1,2}(P)$. There are $n-k$ pairs. As stated in Section 2, a $n$-dimensional arrangement graph contains $n$ subarrangements that we use to derive the desired spanning trees. Let $R$ and $P$ are one of these;
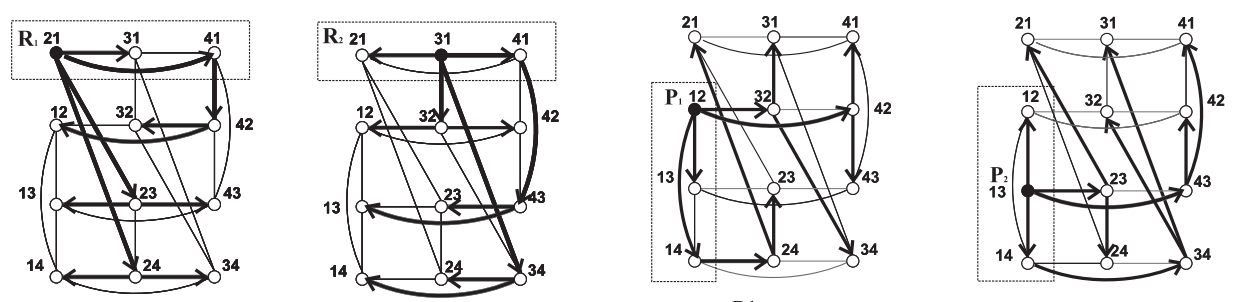

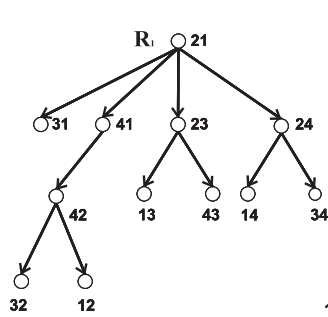

(a)

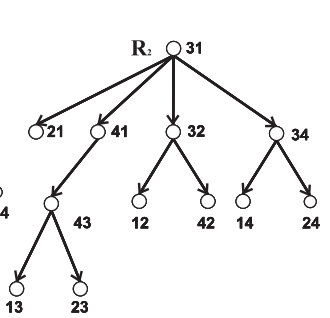

(b)

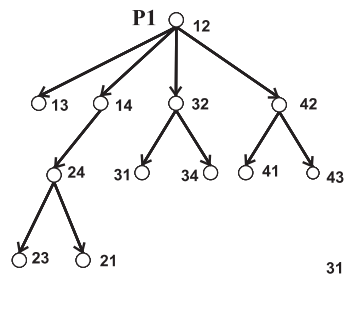

(c)

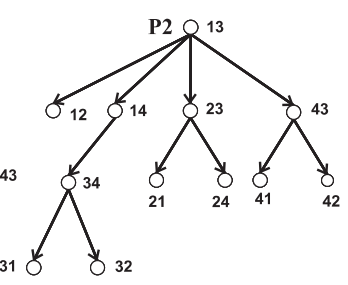

(d)

Fig. 2. Four base edge-disjoint spanning trees in an $A_{4,2}$. 


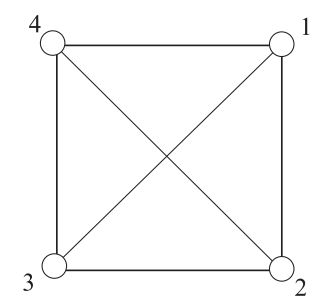

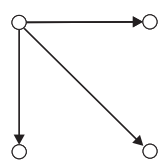

(a)

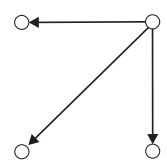

(b)

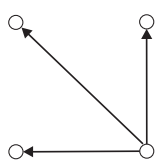

(c)

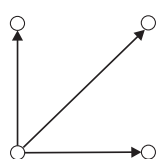

(d)

Fig. 3. Four spanning trees with height 1 in a 4-node complete graph.

we must connect $R$ and $P$ to other $n-1$ subarrangements. For each of $R$ and $P$, we use a single edge to connect the $R$ and $P$ to $n-k$ of subarrangements. We use an intermediate node in same subarrangement as the bridge node to connect $R$ and $P$ to the remaining $k-1$ subarrangements by two edges. If $k=2$, then there is only one subarrangement connecting by two edges.

Rules A1 and $\mathbf{A 2}$ formalize our basic spanning tree construction.

A1 (Single edge). Let nodes $\dot{R}$ and $\dot{P}$ denote two nodes in every $n-k$ subarrangements.

$\left\{\begin{array}{l}\dot{R}=A D J_{2, \alpha}(R), \text { where } \alpha \in E X T(R) . \\ \dot{P}=A D J_{2, \alpha}(P), \text { where } \alpha \in E X T(P) .\end{array}\right.$

Note that $\dot{P}=S W P_{1,2}(\dot{R})$.

A2 (Two edges). Let nodes $\ddot{R}$ and $\ddot{P}$ denote as two nodes in one of remaining $k-1$ subarrangements, which are connected by two edges (Note that in this case, $k=2$ ). Recall in phase 1 , nodes $R_{n-k+1}$ and $P_{n-k+1}$ are the intermediate nodes for nodes $R$ and $P$, respectively. Note that $R_{n-k+1}=S W P_{1,2}\left(P_{n-k+1}\right)$.

$\left\{\ddot{R}=A D J_{2, \beta}\left(R_{n-k+1}\right)\right.$, where $\beta$ is the first bit value in $R$.

$\ddot{P}=A D J_{2, \beta}\left(P_{n-k+1}\right)$, where $\beta$ is the second bit value in $P$.

Note that $\ddot{P}=S W P_{1,2}(\ddot{R})$. Fig. 2(a) shows that root node 21 uses distinct single edge to connecting nodes 23 and 24, and connecting node 42 by two edges. Fig. 2(b) displays that root node 31 directly connects to 32 and 34 but connects 43 by two edges. Fig. 2(c) gives example for other root nodes 12 and 13.

In the following, we describe the existence of $2(n-k)$ base spanning trees. Some notations are defined first. Given root nodes $R_{i}$ and $P_{i}, 1 \leqslant i \leqslant n-k$, are constructed by phase 1 . An $A_{n-k+2,2}$ can be partitioned into $n-k+2$ copies of $A_{n-k+1,1}$ or $A_{n-k+1,1}^{\prime}$ along dimension two or one. Firstly, assume that an $A_{n-k+2,2}$ is partitioned into $n-k+2$ copies of $A_{n-k+1,1}$ along second dimension, where $R_{i}, 1 \leqslant i \leqslant n-k$, located in one of $A_{n-k+1,1}$. Let $I E\left(R_{i}\right), 1 \leqslant i \leqslant n-k$, denote a set of all possible internal edges within each $A_{n-k+1,1}$. Let $E E\left(R_{i}\right)$ denote a set of all possible external edges outgoing each $A_{n-k+1,1}$. Secondly, an $A_{n-k+2,2}$ is partitioned into $n-k+2$ copies of $A_{n-k+1,1}^{\prime}$ along first dimension, where $P_{i}, 1 \leqslant i \leqslant n-k$, located in one of $A_{n-k+1,1}^{\prime}$. Let $I E\left(P_{i}\right), 1 \leqslant i \leqslant n-k$, denote a set of all possible internal edges within each $A_{n-k+1,1}^{\prime}$. Let $E E\left(P_{i}\right)$ denote a set 
of all possible external edges outgoing each $A_{n-k+1,1}^{\prime}$. Further, let $E(x, y)$ denote as edge beginning from node $x$ to destination node $y$. Note that $E(x, y)$ and $E(y, x)$ represent different edges since the link is assumed as full duplex. For example, as illustrated in Fig. 4(a), all bold edges are $I E\left(R_{i}\right)$ and all dash edges are $E E\left(R_{i}\right)$. In Fig. 4(b), all bold edges are $I E\left(P_{i}\right)$ and all dash edges are $E E\left(P_{i}\right)$.

Some important properties are used later as stated herein. For $1 \leqslant i \leqslant n-k$, we have following properties:

P1. Edges in $I E\left(R_{i}\right)$ are equal to edges in $E E\left(P_{i}\right)$.

Fig. 4(a) shows that $E(21,31) \in I E\left(R_{i}\right)$ and $E(21,31) \in E E\left(P_{i}\right)$.

P2. Edges in $I E\left(P_{i}\right)$ are equal to edges in $E E\left(R_{i}\right)$.

Fig. 4(b) displays that $E(21,23) \in I E\left(P_{i}\right)$ and $E(21,23) \in E E\left(R_{i}\right)$.

P3. Edges in $I E\left(R_{i}\right)$ and edges in $E E\left(R_{i}\right)$ are equal to all edges in the $A_{n-k+2,2}$.

P4. Edges in $I E\left(R_{i}\right)$ and edges in $E E\left(R_{i}\right)$ are distinct.

P5. Edges in $I E\left(P_{i}\right)$ and edges in $E E\left(P_{i}\right)$ are distinct.

Lemma 2. There exist $n-k$ edge-disjoint spanning trees $S T_{n-k+2,2}\left(R_{i}\right)$ in an $A_{n-k+2,2}$, for $1 \leqslant i \leqslant n-k$.

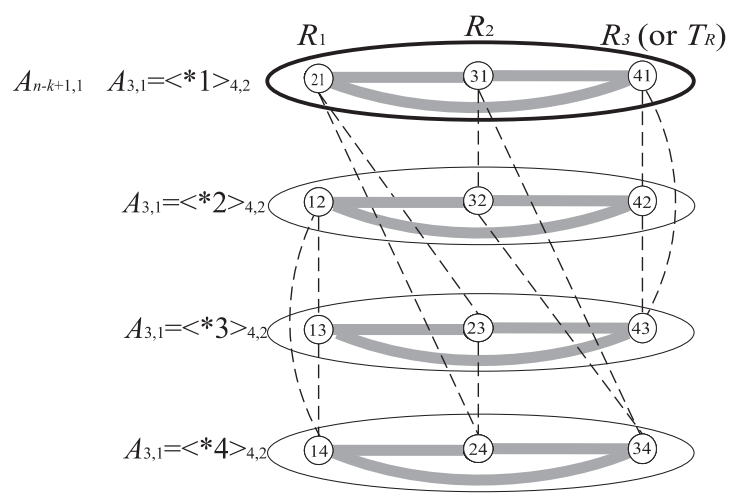

(a)

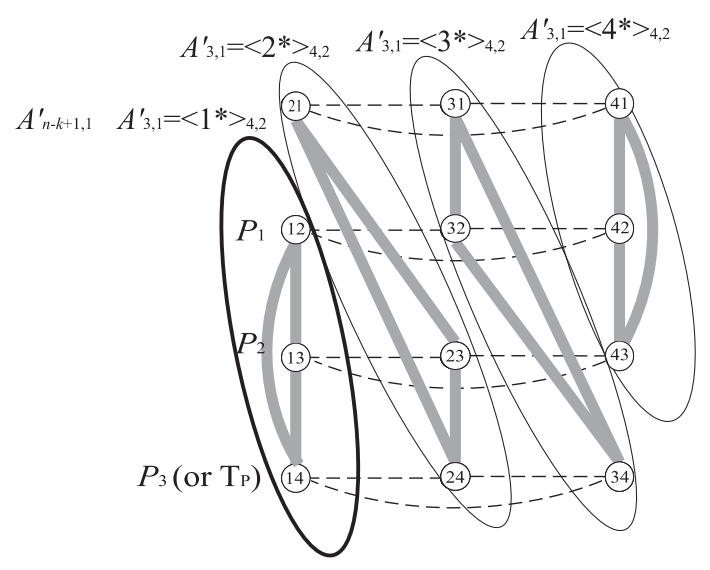

(b)

Fig. 4. Edge distribution in an $A_{4,2}$ : (a) all internal edges within each $A_{n-k+1,1}$ in $A_{4,2}$; (b) all internal edges within each $A_{n-k+1,1}^{\prime}$ in $A_{4,2}$. 
Proof. Given root nodes $R_{i}, 1 \leqslant i \leqslant n-k$, are located in one of partitioned $A_{n-k+1,1}$. Every root node needs to connect to other $A_{n-k+1,1}$. Therefore, $n-k$ spanning trees $S T_{n-k+2,2}\left(R_{i}\right)$, for $1 \leqslant i \leqslant n-k$, are mutually disjoint due to the fact that every root node satisfies the following conditions:

1. All edges of $R_{1}, R_{2}, \ldots$, and $R_{n-k}$ connecting to the same $A_{n-k+1,1}$ are disjoint.

2. All nodes in the same $A_{n-k+1,1}$ connecting to $R_{1}, R_{2}, \ldots$, and $R_{n-k}$ differ from each other.

The reason is stated as follows: recalled again, since $k=2$, for all $R_{i}$, we use $n-2$ distinct single edges connecting to $n-2$ copies of $A_{n-k+1,1}$ and use two edge to connect with remaining one $A_{n-k+1,1}$. Intuitively, all edges connecting from $R_{1}, R_{2}, \ldots$, and $R_{n-k}$ to template node $R_{n-k+1}$ are distinct since all of these nodes located in a $(n-k+1)$-node complete graph (or $\left.A_{n-k+1,1}\right)$. Recall previous notation, edges in all possible $A_{n-k+1,1}$ are denoted as $I E\left(R_{i}\right)$ and all edges in all possible $A_{n-k+1,1}^{\prime}$ are denoted as $E E\left(R_{i}\right)$. For condition 1, all edges of $R_{1}, R_{2}, \ldots$, and $R_{n-k}$ connecting to the same $A_{n-k+1,1}$ are disjoint because every edge belongs to different $A_{n-k+1,1}^{\prime}$. Remember, these edges are belong to $E E\left(R_{i}\right)$. For condition 2, all nodes in the same $A_{n-k+1,1}$ connecting to $R_{1}, R_{2}, \ldots$, and $R_{n-k}$ differ from each other due to the fact that each of these nodes belongs to distinct $A_{n-k+1,1}^{\prime}$.

Based on node symmetry, we have the following similar result.

Lemma 3. There exist $n-k$ edge-disjoint spanning trees $S T_{n-k+2,2}\left(P_{i}\right)$ in an $A_{n-k+2,2}$, for $1 \leqslant i \leqslant n-k$.

To make the clear description of lemma 6, some notations are defined. Let $S E\left(R_{i}, A\right), A \in A_{n-k+1,1}$ or $A_{n-k+1,1}^{\prime}$, denote a set of spanning edges which exist in tree $S T\left(R_{i}\right)$ belonging to subarrangement $A$. In the same way, $S E\left(P_{i}, A\right), A \in A_{n-k+1,1}$ or $A_{n-k+1,1}^{\prime}$, denote a set of spanning edges which exist in tree $S T\left(P_{i}\right)$ belonging to subarrangement $A$. For example in Fig. 4(b), edges $E(21,23)$ and $E(21,24)$ belong to $S E(21,\langle 2 *\rangle) \in E E(21)$. Edges $E(24,21)$ and $E(24,23)$ belong to $S E(12,\langle 2 *\rangle) \in I E(12)$.

Two other important properties are stated. For $1 \leqslant i \leqslant n-k$, we have the following properties:

P6. $\quad\left\{\right.$ Spanning edges in tree $S T\left(R_{i}\right)$ belonging to $A_{n-k+1,1}$, i.e., $S E\left(R_{i}, A_{n-k+1,1}\right), \in I E\left(R_{i}\right)$ Spanning edges in tree $S T\left(R_{i}\right)$ belonging to $A_{n-k+1,1}^{\prime}$, i.e., $S E\left(R_{i}, A_{n-k+1,1}^{\prime}\right), \in E E\left(R_{i}\right)$

For instance as shown in Fig. 4(a) and (b), $E(24,14) \in S E\left(21,\langle * 4\rangle_{4,2}\right) \in I E(21)$ and $E(21,24)$ $\in \operatorname{SE}\left(21,\langle 2 *\rangle_{4,2}\right) \in E E(21)$.

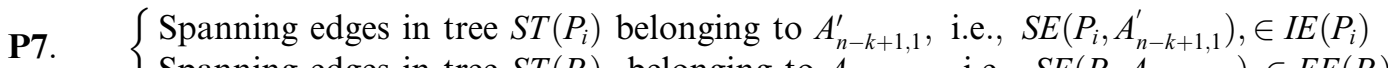
Spanning edges in tree $S T\left(P_{i}\right)$ belonging to $A_{n-k+1,1}$, i.e., $S E\left(P_{i}, A_{n-k+1,1}\right), \in E E\left(P_{i}\right)$

For instance as illustrated in Fig. 4(b) and (a), $E(24,21) \in S E\left(12,\langle 2 *\rangle_{4,2}\right) \in \operatorname{IE}(12)$ and $E(14,24) \in S E\left(12,\langle * 4\rangle_{4,2}\right) \in E E(12)$.

Lemma 4. Each pair of $S T_{n-k+2,2}\left(R_{i}\right)$ and $S T_{n-k+2,2}\left(P_{i}\right)$, for $1 \leqslant i \leqslant n-k$, are mutually disjoint.

Proof. Initially, all edges of root nodes $R_{i}$ connecting to template node $R_{n-k+1}$ located in one $(n-k+1)$ node complete graph, namely $\hat{A}_{n-k+1,1}$, and all edges of root nodes $P_{i}$ connecting to template node $P_{n-k+1}$ located in another one $(n-k+1)$-node complete graph, namely $\hat{A}_{n-k+1,1}^{\prime}$. Note that, all spanning trees $S T_{n-k+2,2}\left(R_{i}\right)$ do not use any internal edge of $\hat{A}_{n-k+1,1}^{\prime}$ and all spanning trees $S T_{n-k+2,2}\left(P_{i}\right)$ do not use any internal edge of $\hat{A}_{n-k+1,1}$. For example as shown in Fig. 4, edges for $R_{1}$ and $R_{2}$ connecting to template node $R_{n-k+1}$ belong to a complete graph $A_{n-k+1,1}=A_{3,1}=\langle * 1\rangle_{4,2}$. And edges for $P_{1}$ and $P_{2}$ connecting to template node $P_{n-k+1}$ belong to another complete graph $A_{n-k+1,1}^{\prime}=A_{3,1}^{\prime}=\langle 1 *\rangle_{4,2}$. Clearly, all of these edges are disjoint. Based on $\mathbf{P 1}$ and $\mathbf{P 2}$ property, for all $S T_{n-k+2,2}\left(R_{i}\right)$ and $S T_{n-k+2,2}\left(P_{i}\right), 1 \leqslant i \leqslant n-k$, may spread its subtree in same subarrangement $A_{n-k+1,1}^{\prime}$ or $A_{n-k+1,1}$. For an $A_{n-k+1,1}$ containing root node $R_{i}, 1 \leqslant i \leqslant n-k$, 
using $n-1$ edges connecting to other $A_{n-k+1,1}$ denote as $\operatorname{SE}\left(R_{i}, A_{n-k+1,1}^{\prime}\right) \in E E\left(R_{i}\right)=\operatorname{IE}\left(P_{i}\right)$. Each pair of $S T_{n-k+2,2}\left(R_{i}\right)$ and $S T_{n-k+2,2}\left(P_{i}\right)$, for $1 \leqslant i \leqslant n-k$, are said to be mutually disjoint if and only if we can prove that $S E\left(R_{i}, A_{n-k+1,1}^{\prime}\right)$ and $S E\left(P_{i}, A_{n-k+1,1}^{\prime}\right), 1 \leqslant i \leqslant n-k$ are edge-disjoint and $S E\left(R_{i}, A_{n-k+1,1}\right)$ and $\operatorname{SE}\left(P_{i}, A_{n-k+1,1}\right), 1 \leqslant i \leqslant n-k$, are edge-disjoint. These two cases are stated as follows:

(1) Initially, we show that all of spanning tree edges in tree $S T\left(R_{i}\right)$ and $S T\left(P_{i}\right)$ belonging to same subarrangement $A_{n-k+1,1}^{\prime}$, i.e., $S E\left(R_{i}, A_{n-k+1,1}^{\prime}\right)$ and $S E\left(P_{i}, A_{n-k+1,1}^{\prime}\right), 1 \leqslant i \leqslant n-k$, are edge-disjoint. Note that, $S E\left(R_{i}, A_{n-k+1,1}^{\prime}\right) \in E E\left(R_{i}\right)=I E\left(P_{i}\right)$ and $S E\left(P_{i}, A_{n-k+1,1}^{\prime}\right) \in I E\left(P_{i}\right)=E E\left(R_{i}\right)$. The reason of $S E\left(R_{i}, A_{n-k+1,1}^{\prime}\right)$ and $\operatorname{SE}\left(P_{i}, A_{n-k+1,1}^{\prime \prime}\right)$ being edge-disjoint is stated as follows. For every spanning tree $S T_{n-k+2,2}\left(P_{i}\right)$ may spread its subtree in same $A_{n-k+1,1}^{\prime}$ beginning from a node, namely first node. $S E\left(R_{i}, A_{n-k+1,1}^{\prime}\right)$ and $S E\left(P_{i}, A_{n-k+1,1}^{\prime}\right)$ are edge-disjoint if all of first nodes are different in same $A_{n-k+1,1}^{\prime}$. In every same $A_{n-k+1,1}^{\prime}, S E\left(R_{i}, A_{n-k+1,1}^{\prime}\right) \in$ $E E\left(R_{i}\right)=\operatorname{IE}\left(P_{i}\right)$ and $\operatorname{SE}\left(P_{i}, A_{n-k+1,1}^{\prime}\right) \in I E\left(P_{i}\right)=E E\left(R_{i}\right), 1 \leqslant i \leqslant n-k$, are edge-disjoint because the corresponding first node is different. This is because that all first nodes are located in different $A_{n-k+1,1}$.

(2) Next, we prove that all of spanning tree edges in tree $S T\left(R_{i}\right)$ and $S T\left(P_{i}\right)$ belonging to same subarrangement $A_{n-k+1,1}$, i.e, $S E\left(R_{i}, A_{n-k+1,1}\right)$ and $S E\left(P_{i}, A_{n-k+1,1}\right), 1 \leqslant i \leqslant n-k$, are edge-disjoint. Note that $S E\left(R_{i}, A_{n-k+1,1}\right) \in I E\left(R_{i}\right)=E E\left(P_{i}\right)$ and $S E\left(P_{i}, A_{n-k+1,1}\right) \in E E\left(P_{i}\right)=I E\left(R_{i}\right)$. Due to node symmetry, so we omit the detail, but $S E\left(R_{i}, A_{n-k+1,1}\right)$ and $S E\left(P_{i}, A_{n-k+1,1}\right), 1 \leqslant i \leqslant n-k$ are edge-disjoint in the same $A_{n-k+1,1}$ by the similar reason in the case 1 .

Fig. 4 illustrates an example that let $R_{1}=21$, it has two direct edges $S E\left(21,\langle 2 *\rangle_{4,2}\right) \in E E(21)$ connecting to subarrangement $\langle * 3\rangle_{4,2},\langle * 4\rangle_{4,2}=A_{3,1}$ with nodes 23,24 and one edge $S E\left(21,\langle 4 *\rangle_{4,2}\right) \in E E(21)$ for node 41 connecting to another subarrangement $\langle * 2\rangle_{4,2}=A_{3,1}$ with node 42 . Spanning tree $S T_{4,2}\left(R_{1}\right), S T_{4,2}\left(P_{1}\right)$ and $S T_{4,2}\left(P_{2}\right)$, respectively spread their subtrees in $\langle 2 *\rangle_{4,2}=A_{3,1}^{\prime}$ by first node $21,24,23$ and spread subtrees in $\langle 4 *\rangle_{4,2}=A_{3,1}^{\prime}$ by first node $41,42,43$. We can find that the edges for all spanning trees spread its subtrees in subarrangement $\langle 2 *\rangle_{4,2}=A_{3,1}^{\prime}$ and $\langle 4 *\rangle_{4,2}=A_{3,1}^{\prime}$ are disjoint from each other due to the fact that all the first nodes are different and located in different $A_{3,1}$.

Theroem 1. There exist $2(n-k)$ base edge-disjoint spanning trees $S T_{n-k+2,2}\left(R_{i}\right)$ and $S T_{n-k+2,2}\left(P_{i}\right)$ in an $A_{n-k+2,2}$, where $1 \leqslant i \leqslant n-k$. Each spanning tree's height is 3 .

Proof. Given root nodes $R_{i}$ and $P_{i}$, where $1 \leqslant i \leqslant n-k$. First, there exist $n-k$ spanning trees $S T_{n-k+2,2}\left(R_{i}\right)$ and $S T_{n-k+2,2}\left(P_{i}\right)$ in an $A_{n-k+2,2}$, for $1 \leqslant i \leqslant n-k$, in Lemmas 2 and 3. Lemma 4 illustrates that each pair of $S T_{n-k+2,2}\left(R_{i}\right)$ and $S T_{n-k+2,2}\left(P_{i}\right)$, for $1 \leqslant i \leqslant n-k$, are mutually disjoint. Hence the theorem.

\subsection{Phase 2. Construction of $2(n-k)$ edge-disjoint spanning trees in an $A_{n, k}$}

The above section constructed $2(n-k)$ base spanning trees in $A_{n-k+2,2}$, and each base spanning tree's height is 3 . In this section, we describe how to construct $2(n-k)$ spanning trees $S T_{n, k}\left(R_{i}\right)$ and $S T_{n, k}\left(P_{i}\right)$, for $1 \leqslant i \leqslant n-k$ in an $A_{n, k}$, where the height of each spanning tree is $2 k-1$.

For induction, we construct $2(n-k)$ spanning trees in an $A_{n, k}$ by using $2 n(n-k)$ spanning tress in $n$ copies of $A_{n-1, k-1}$. Our major task is to connect $2 n(n-k)$ spanning subtrees into $2(n-k)$ spanning trees $S T_{n, k}\left(R_{i}\right)$ and $S T_{n, k}\left(P_{i}\right), 1 \leqslant i \leqslant n-k$.

Consider an $A_{n, k}$ which is partitioned into $n$ copies of $A_{n-1, k-1}$. Assume that $2(n-k)$ spanning trees $S T_{n-1, k-1}\left(R_{i}\right)$ and $S T_{n-1, k-1}\left(P_{i}\right), i=1, \ldots, n-k$, can be constructed in each $A_{n-1, k-1}$ if their root nodes satisfied the root-location property. A randomly selected $A_{n-1, k-1}$ serves as beginning spanning tree. From this $A_{n-1, k-1}$, there exist $2(n-k)$ spanning trees $S T_{n-1, k-1}\left(R_{i}\right)$ and $S T_{n-1, k-1}\left(P_{i}\right), 1 \leqslant i \leqslant n-k$, where $R_{i}$ and $P_{i}$ are root nodes. Let root nodes $R_{i}$ and $P_{i}$ connect to root nodes $R_{i}^{\prime}$ and $P_{i}^{\prime}$, where $R_{i}^{\prime}$ and $P_{i}^{\prime}$ located in other $A_{n-1, k-1}$. If $R_{i}^{\prime}$ and $P_{i}^{\prime}$ satisfy the root-location property, therefore we can embed $2(n-k)$ spanning trees 
$S T_{n, k}\left(R_{i}\right)$ and $S T_{n, k}\left(P_{i}\right), 1 \leqslant i \leqslant n-k$, on each of the $A_{n-1, k-1}$, so we can recursively construct $2(n-k)$ spanning trees in an $A_{n, k}$.

Given $2(n-k)$ root nodes $R_{i}$ and $P_{i}, 1 \leqslant i \leqslant n-k$. Consider any pair of nodes $R \in R_{i}$ and $P \in P_{i}$, where $R=S W P_{1,2}(P)$. Note that there are $n-k$ pairs. A $n$-dimensional arrangement graph contains $n$ subarrangements that we use to derive the desired spanning trees. Let $R$ and $P$ are one of these; we must connect $R$ and $P$ to other $n-1$ subarrangements. For each of $R$ and $P$, we use a single edge to connect the $R$ and $P$ to $n-k$ of subarrangements. We also use an intermediate node in same subarrangement as the bridge node to connect $R$ and $P$ to the remaining $k-1$ subarrangements by two edges.

Rules $\mathbf{A 1} \mathbf{1}^{\prime}$ and $\mathbf{A 2} \mathbf{2}^{\prime}$ formalize our recursively spanning tree construction.

$\mathbf{A 1}^{\prime}$ (Single edge). The part is same as A1. Let nodes $\dot{R}$ and $\dot{P}$ denote two nodes in every $n-k$ subarrangement.

$\left\{\begin{array}{l}\dot{R}=A D J_{2, \alpha}(R), \text { where } \alpha \in E X T(R) . \\ \dot{P}=A D J_{2, \alpha}(P), \text { where } \alpha \in E X T(P) .\end{array}\right.$

Note that $\dot{P}=S W P_{1,2}(\dot{R})$.

$\mathbf{A 2}^{\prime}$ (Two edges). This part is same as A2. Let nodes $\ddot{R}$ and $\ddot{P}$ denote as two nodes in one of remaining $k-1$ subarrangements which connected by two edges. Nodes $R_{n-k+1}$ and $P_{n-k+1}$ are the intermediate nodes for nodes $R$ and $P$, respectively. Note that $R_{n-k+1}=S W P_{1,2}\left(P_{n-k+1}\right)$.

$\left\{\ddot{R}=A D J_{2, \beta}\left(R_{n-k+1}\right)\right.$, where $\beta$ is the first bit value in $R$.

$\left\{\ddot{P}=A D J_{2, \beta}\left(P_{n-k+1}\right)\right.$, where $\beta$ is the second bit value in $P$.

Note that $\ddot{P}=S W P_{1,2}(\ddot{R})$. (See example in Fig. 5).

Assume that $R_{i}^{\prime}$ and $P_{i}^{\prime}, 1 \leqslant i \leqslant n-k$, are new connecting root nodes in each of other $(n-1)$-subarrangement. Ensuring that root nodes $R_{i}^{\prime}$ and $P_{i}^{\prime}$ satisfied the root-location property would allow us to establish our spanning trees $S T_{n, k}\left(R_{i}\right)$ and $S T_{n, k}\left(P_{i}\right)$, for $i=1, \ldots, n-k$. In the following, we show the correctness that root nodes $R_{i}^{\prime}$ and $P_{i}^{\prime}$ satisfied root-location property, for $i=1, \ldots, n-k$.

Lemma 5. There are $2(n-k)$ root nodes $R_{i}^{\prime}$ and $P_{i}^{\prime}, 1 \leqslant i \leqslant n-k$, in same subarrangement graph and satisfy the root-location property.

Proof. Without loss of generality, assume that $n-k$ root nodes $R_{i}, 1 \leqslant i \leqslant n-k$, represented as $\left(a b y_{3} y_{4} \cdots y_{k}\right) \in\left\langle * * y_{3} y_{4} \cdots y_{k}\right\rangle_{n, k}$ and $P_{i}, 1 \leqslant i \leqslant n-k$, represented as $\left(b a y_{3} y_{4} \cdots y_{k}\right) \in\left\langle * * y_{3} y_{4} \cdots y_{k}\right\rangle_{n, k}, R_{i}$ and $P_{i}$ connect to $R_{i}^{\prime}$ and $P_{i}^{\prime}, 1 \leqslant i \leqslant n-k$, respectively. Our results demonstrate that root nodes $R_{i}^{\prime}$ and $P_{i}^{\prime}$, $1 \leqslant i \leqslant n-k$, in same subarrangement graph and satisfy the root-location property. Three possible cases are discussed.

(1) (Both links are single edge) Since $\operatorname{EXT}\left(R_{i}=\left(a b y_{3} y_{4}, \ldots, y_{k}\right)\right)=\operatorname{EXT}\left(P_{i}=\left(b a y_{3} y_{4} \cdots y_{k}\right)\right)$, so it is possible to let $R_{i}^{\prime}=\left(a b y_{3} y_{4} \cdots y_{k-1} \gamma\right)$ and $P_{i}^{\prime}=\left(b a y_{3} y_{4} \cdots y_{k-1} \gamma\right)$, where $\gamma$ belongs to a set of elements which are not used in all root nodes $R_{1}, \ldots$, and $R_{n-k}$ or $P_{1}, \ldots$, and $P_{n-k}$, which can be represented as $\gamma \in \operatorname{EXT}\left(R_{1}\right) \cap \operatorname{EXT}\left(R_{2}\right) \cap \cdots \cap \operatorname{EXT}\left(R_{n-k}\right)=E X T\left(P_{1}\right) \cap \operatorname{EXT}\left(P_{2}\right) \cap, \ldots, \cap E X T\left(P_{n-k}\right)$. Obviously, $P_{i}^{\prime}=S W P_{1,2}$ $\left(R_{i}^{\prime}\right) \in\left\langle * * y_{3} y_{4} \cdots y_{k-1} \gamma\right\rangle_{n, k}$ satisfies the root-location property. For instance, $215 \rightarrow 214,125 \rightarrow 124$, $315 \rightarrow 314$, and $135 \rightarrow 134$, where $214=S W P_{1,2}(124)$ and $314=S W P_{1,2}(134)$.

(2) (One link is single link, other link is two edges) For $1 \leqslant i \neq j \leqslant n-k$, let $R_{i}=\left(\alpha b y_{3} y_{4} \cdots y_{k}\right), R_{j}=$ $\left(\beta b y_{3} y_{4} \cdots y_{k}\right), P_{i}=\left(b \alpha y_{3} y_{4} \cdots y_{k}\right)$, and $P_{j}=\left(b \beta y_{3} y_{4} \cdots y_{k}\right) \in\left\langle * * y_{3} y_{4} \cdots y_{k}\right\rangle_{n, k}$. Note that $P_{i}=S W P_{1,2}\left(R_{i}\right)$, $P_{j}=S W P_{1,2}\left(R_{j}\right)$. First, if $\beta \in E X T\left(R_{i}\right)=E X T\left(P_{i}\right)$, then $R_{i}^{\prime}=\left(\alpha b y_{3} y_{4} \cdots y_{k-1} \beta\right)$ and $P_{i}^{\prime}=\left(b \alpha y_{3} y_{4} \cdots y_{k-1} \beta\right)$. Second, if $\beta \in \operatorname{INT}\left(R_{j}\right)=\operatorname{INT}\left(P_{j}\right)$, two edges are needed for $R_{j}$ and $P_{j}$ to connect $R_{j}^{\prime}$ and $P_{j}^{\prime}$ in subarrangement $\left\langle * * y_{3} y_{4} \cdots y_{k-1} \beta\right\rangle_{n, k}$, respectively. Then let $\omega \in E X T\left(R_{j}\right)$, we can obtain $R_{j}=\left(\beta b y_{3} y_{4} \cdots y_{k}\right) \rightarrow$ $\left(\omega b y_{3} y_{4} \cdots y_{k}\right) \rightarrow\left(\omega b y_{3} y_{4} \cdots y_{k-1} \beta\right)=R_{j}^{\prime}$ and $P_{j}=\left(b \beta y_{3} y_{4} \cdots y_{k}\right) \rightarrow\left(b \omega y_{3} y_{4} \cdots y_{k}\right) \rightarrow\left(b \omega y_{3} y_{4} \cdots y_{k-1} \beta\right)=P_{j}^{\prime}$. 

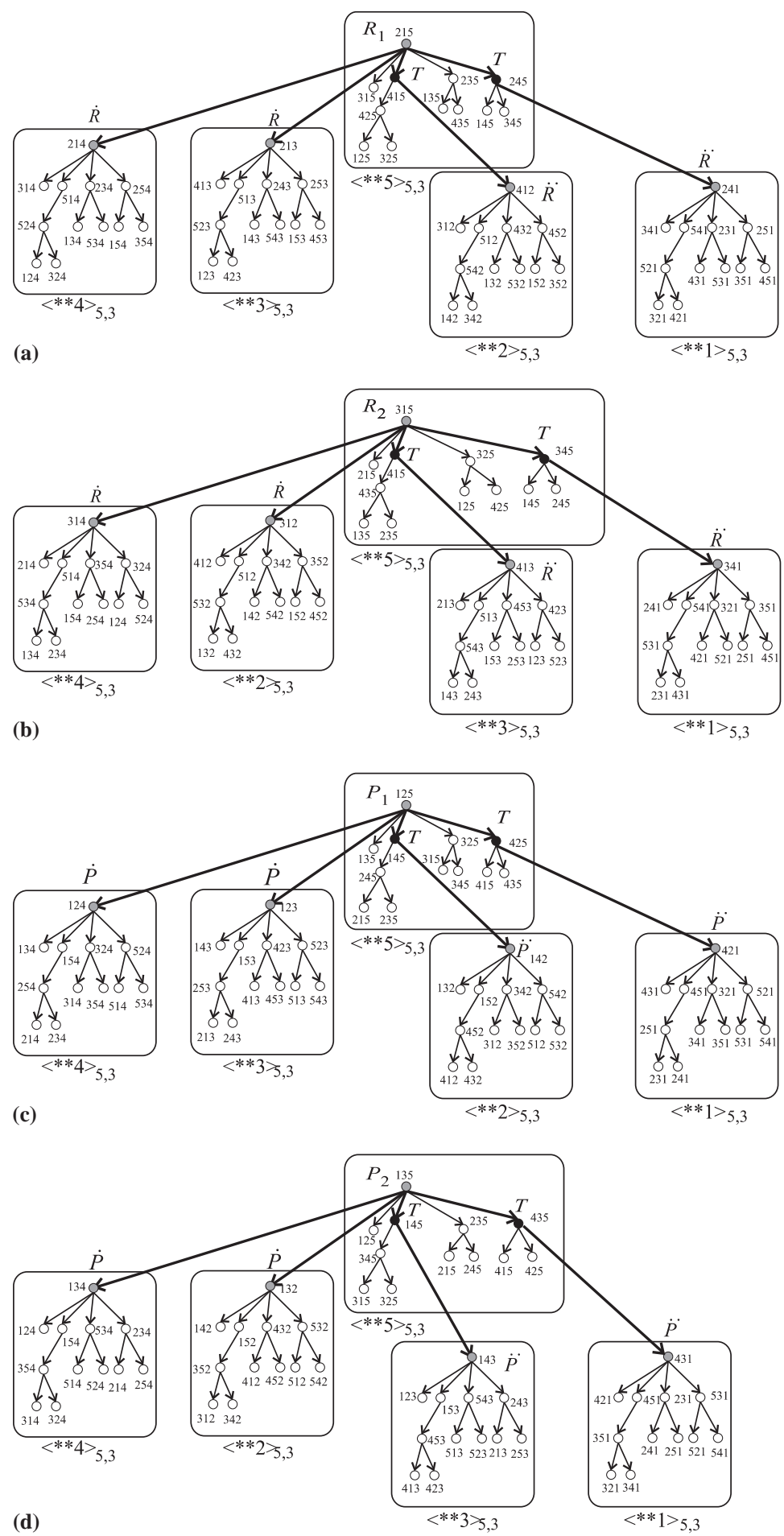

Fig. 5. Example of four spanning trees in an $A_{5,3}$ : (a) from root node 215; (b) from root node 315; (c) from root node 125; (d) from root node 135 . 

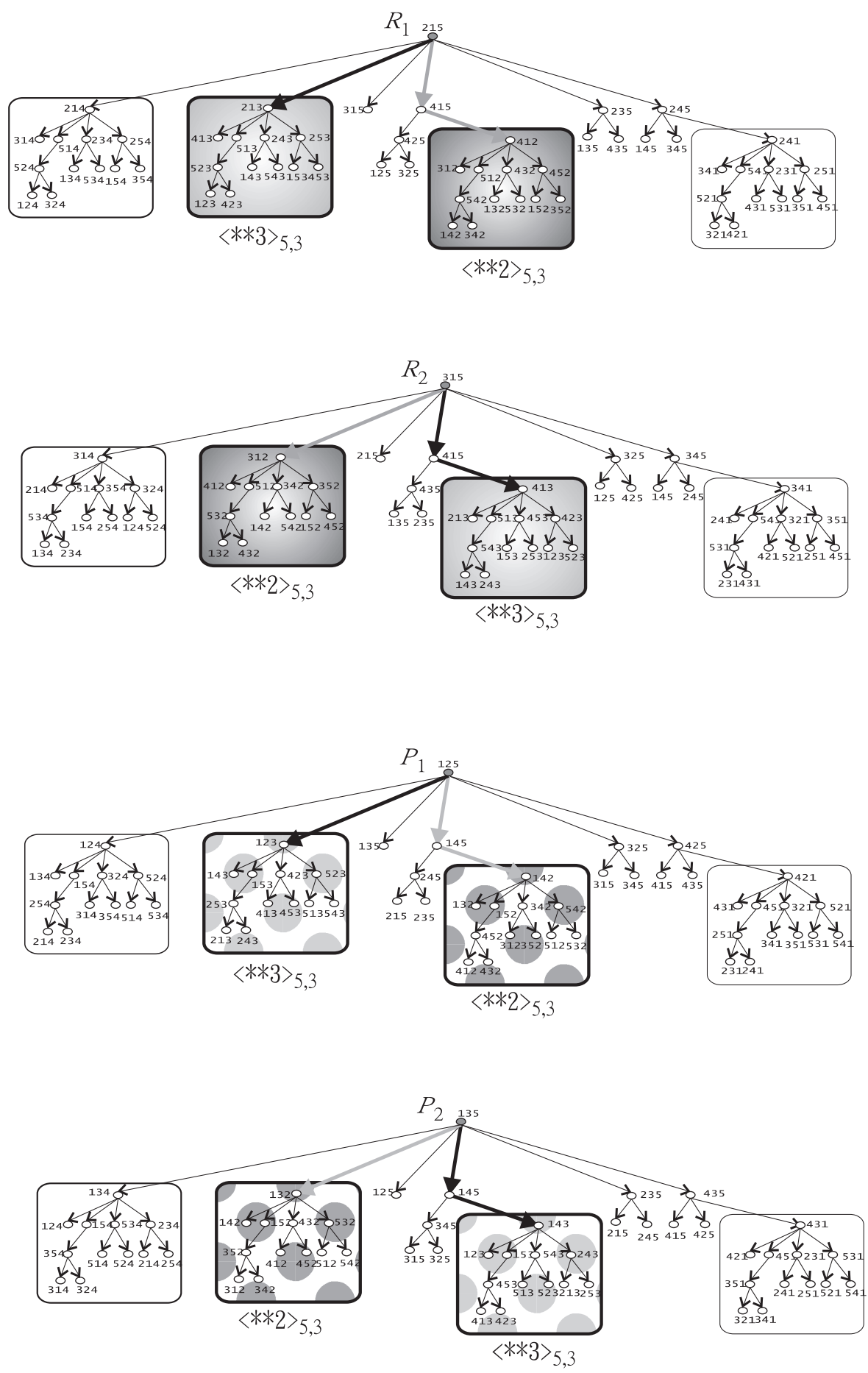

Fig. 6. Example of proof in Lemma 5. 
Therefore, $P_{i}^{\prime}=\operatorname{SWP}_{1,2}\left(R_{i}^{\prime}\right)$ and $P_{j}^{\prime}=S W P_{1,2}\left(R_{j}^{\prime}\right) \in\left\langle * * y_{3} y_{4} \cdots y_{k-1} \beta\right\rangle_{n, k}$ satisfy the root-location property. Fig. 6 provides an example that $215 \rightarrow 213,125 \rightarrow 123,315 \rightarrow 415 \rightarrow 413$, and $135 \rightarrow 145 \rightarrow 143$, where $213=S W P_{1,2}(123)$ and $413=S W P_{1,2}(143)$.

(3) (Both links are two edges) For $1 \leqslant i \leqslant n-k$, let $R_{i}=\left(a b y_{3} y_{4} \cdots y_{k}\right)$ and $P_{i}=\left(b a y_{3} y_{4} \cdots y_{k}\right)$. Due to $b \in I N T\left(R_{i}\right)=I N T\left(P_{i}\right)$, two edges are needed for $R_{i}$ and $P_{i}$ to connect $R_{i}^{\prime}$ and $P_{i}^{\prime}$ in subarrangement $\left\langle * * y_{3} y_{4} \cdots y_{k-1} b\right\rangle_{n, k}$, respectively. Without loss of generality, if $\gamma$ belongs to a set of elements which are not used in root nodes $R_{1}, \ldots$, and $R_{n-k}$, we can obtain that $R_{i}=\left(a b y_{3} y_{4} \cdots y_{k}\right) \rightarrow\left(a \gamma y_{3} y_{4} \cdots y_{k}\right) \rightarrow$ $\left(a \gamma y_{3} y_{4} \cdots y_{k-1} b\right)=R_{i}^{\prime}$, and $P_{i}=\left(\right.$ bay $\left._{3} y_{4} \cdots y_{k}\right) \rightarrow\left(\gamma a y_{3} y_{4} \cdots y_{k}\right) \rightarrow\left(\gamma a y_{3} y_{4} \cdots y_{k-1} b\right)=P_{i}^{\prime}$. Therefore, $P_{i}^{\prime}=$ $S W P_{1,2}\left(R_{i}^{\prime}\right) \in\left\langle * * y_{3} y_{4} \cdots y_{k-1} b\right\rangle_{n, k}$ satisfies the root-location property. For example, $215 \rightarrow 245 \rightarrow 241$, $125 \rightarrow 425 \rightarrow 421,315 \rightarrow 345 \rightarrow 341, \quad$ and $\quad 135 \rightarrow 435 \rightarrow 431, \quad$ where $241=S W P_{1,2}(421)$ and $341=S W P_{1,2}(431)$.

Lemma 6. All edges for $R_{i}$ and $P_{i}, 1 \leqslant i \leqslant n-k$, respectively connect to root nodes $R_{i}^{\prime}$ and $P_{i}^{\prime}$ which are located in other subarrangement graphs are all edge-disjoint.

Proof. Note that root nodes $R_{i}$ and $P_{i}, 1 \leqslant i \leqslant n-k$, are all distinct, and $R_{i}$ and $P_{i}$ use same rule to connect with $R_{i}^{\prime}$ and $P_{i}^{\prime}$, therefore $R_{i}$ and $P_{i}$ are also different from each other. Due to different source and destination, all edges for $R_{i}$ and $P_{i}$, respectively connect to root nodes $R_{i}^{\prime}$ and $P_{i}^{\prime}$ which are located in other subarrangement graphs are all edge-disjoint.

Lemma 7. The height of $2(n-k)$ edge disjoint spanning trees $S T_{n, k}\left(R_{i}\right)$ and $S T_{n, k}\left(P_{i}\right)$ in an $A_{n, k}$ is $2 k-1$.

Proof. There are $k$ steps to construct $S T_{n, k}\left(R_{i}\right)$ and $S T_{n, k}\left(P_{i}\right), 1 \leqslant i \leqslant n-k$. Each step needs at most two edges except that the final step in $A_{n-k+1,1}$ needs one single edge since $A_{n-k+1,1}$ is a complete graph. The height is $2(k-1)+1=2 k-1$.

Theroem 2. There exist $2(n-k)$ edge-disjoint spanning trees $S T_{n, k}\left(R_{i}\right)$ and $S T_{n, k}\left(P_{i}\right)$ with height $2 k-1$ in an $A_{n, k}$, where $i=1, \ldots, n-k$.

\section{Conclusions}

In this paper, we consider the problem of congestion-free embedding of multiple spanning trees in an arrangement graph. This is first result to exploit multiple spanning trees. In this paper, we develop a bottom-up congestion-free embedding of $2(n-k)$ spanning trees with height $2 k-1$ in an $(n, k)$-dimensional arrangement graph. A well-known application of edge-disjoint multiple spanning trees is the broadcasting algorithm. Work is currently underway to develop the optimal number of spanning trees in $A_{n, k}$ by exploiting $O(k(n-k))$ edge-disjoint spanning trees.

\section{References}

[1] S.B. Akers, D. Harel, B. Krishnameurthy, The star graph: an attractive alternative to the $n$-cube, in: Proceedings of the International Conference on Parallel Processing, 1987, pp. 393-400.

[2] L.Q. Bai, H. Ebara, H. Nakano, H. Maeda, Fault-tolerant broadcasting on the arrangement graph, Comput J 41 (3) (1998) 171-184.

[3] Y.-S. Chen, T.-J. Juang, E.-H. Tseng, Efficient broadcast in an arrangement graph using multiple spanning trees, IEICE Trans Fundamentals Elect Commun Comput Sci E83-A (1) (2000) 139-149.

[4] Y.-S. Chen, J.-P. Sheu, A fault-tolerant reconfiguration scheme in the faulty star graph, J Inform Sci Eng 16 (1) (2000) $25-40$. 
[5] K. Day, A. Tripathi, Characterization of node disjoint path in arrangement graphs, in: Technical Report TR91-43, Computer Science Department, University of Minnesota, 1991.

[6] K. Day, A. Tripathi, Arrangement grapha: a class of generalized star graphs, Inform Process Lett 42 (5) (1992) $235-241$.

[7] K. Day, A. Tripathi, Embedding cycles in arrangement graphs, IEEE Trans Comput 12 (8) (1992) 1002-1006.

[8] K. Day, A. Tripathi, Embedding grids, hypercube, and trees in arrangement graphs, in: Proceedings of the International Conference on Parallel Processing, 1999, pp. 3-65-3-72.

[9] S.-Y. Hsieh, G.-H. Chen, C.-W. Ho, Fault-free hamiltonian cycles in faulty arrangement graphs, IEEE Trans Parallel Distributed Sys 10 (3) (1999) 223-237.

[10] J.-P. Sheu, C.-T. Wu, T.-S. Chen, An optimal broadcasting algorithm without message redundancy in star graphs, IEEE Trans Parallel Distributed Sys 6 (6) (1995) 653-658.

[11] S.-S. Tsai, S.-J. Horng, Efficient embedding hypercube on arrangement graphs, J Inform Sci Eng 12 (4) (1996) $585-592$.

[12] Y.-C. Tseng, S.-H. Chang, J.-P. Sheu, Fault-tolerant ring embedding in star graphs, IEEE Trans Parallel Distributed Sys 8 (12) (1997) 1185-1195.

[13] Y.-C. Tseng, Y.-S. Chen, T.-Y. Juang, C.-J. Chang, Congestion-free, dilation-2 embeding of complete binary tree in star graphs, Networks 33 (3) (1999) 221-231.

[14] Y.-C. Tseng, J.-P. Sheu, Toward optimal broadcast in a star graph using multiple spanning trees, IEEE Trans Comput 46 (5) (1997) 593-599.

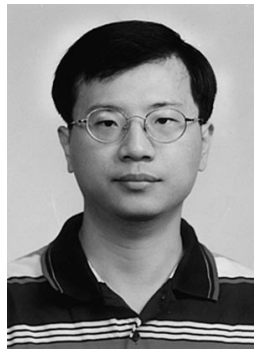

Yuh-Shyan Chen received the B.S. degree in computer science from Tamkang University, Taiwan, Republic of China, in June 1988 and the M.S. and Ph.D. degrees in Computer Science and Information Engineering from the National Central University, Taiwan, Republic of China, in June 1991 and January 1996, respectively. He joined the faculty of Department of Computer Science and Information Engineering at Chung-Hua University, Taiwan, Republic of China, as an associate professor in February 1996. He joined the Department of Statistic, National Taipei University in August 2000. His current research includes parallel/distributed processing, network security, wireless communication/mobile computing, and ad-hoc QoS routing protocols. Dr. Chen is a member of the IEEE Computer Society and Phi Tau Phi Society.

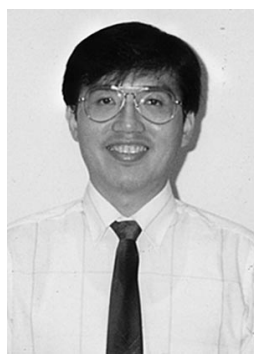

Tong-Ying Juang is an associate professor in the Department of Statistics at National Taipei University. His research interests include distributed and parallel algorithms, fault-tolerant distributed computing, mobile computing, and interconnection networks. He received a B.S. in Naval architecture from National Taiwan University, and his M.S. and Ph.D in computer science from the University of Texas at Dallas 1989 and 1992, respectively. Juang is a member of the IEEE. Contact him at the Department of Statistics, National Taipei University, Taipei, 10433, Taiwan; juang@cc.nchulc.edu.tw

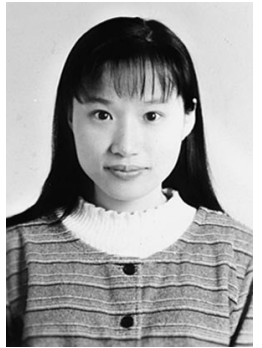

Ying-Ying Shen received the B.S. and M.S. degree in computer science and information engineering from Chung-Hua University, Taiwan, Republic of China, in June 1997 and June 1999, respectively. She joined the Optoma Corporation, Taiwan, ROC, as an ERP Database Administrator in June 1999. Her current research interests include Oracle database, network computing architecture. 